\title{
Topological feature and phase diagram of QCD at complex chemical potential
}

\section{Kouji Kashiwa*}

Yukawa Institute for Theoretical Physics, Kyoto University, Kyoto 606-8502, Japan

E-mail: kouji.kashiwa@yukawa.kyoto-u.ac.jp

\section{Akira Ohnishi}

Yukawa Institute for Theoretical Physics, Kyoto University, Kyoto 606-8502, Japan

E-mail: ohnishi@yukawa.kyoto-u.ac.jp

\begin{abstract}
We propose new determination of the confinement-deconfinement (pseudo) critical temperature by using the analogy of the topological order discussed in the condensed matter physics. The imaginary chemical potential is interpreted as the Aharonov-Bohm phase, then the Roberge-Weiss endpoint would define the confinement-deconfinement (pseudo) critical temperature. Expected QCD phase diagram diagrams are presented at finite complex chemical potential based on the perturbative calculation.
\end{abstract}

The 33rd International Symposium on Lattice Field Theory

14 -18 July 2015

Kobe International Conference Center, Kobe, Japan*

${ }^{*}$ Speaker. 


\section{Introduction}

Understanding of QCD phase diagram at finite temperature $(T)$ and real quark chemical potential $\left(\mu_{\mathrm{R}}\right)$ is one of the interesting and important subjects in the nuclear and elementary particle physics. If we can obtain the QCD phase diagram from the first-principle calculation, there is no unclearness. However, such first principle calculation which is the lattice QCD simulation has the sign problem at finite $\mu_{R}$. Even if several methods to circumvent the sign problem are used, we can not reach the $\mu_{\mathrm{R}} / T>1$ region. Therefore, several effective model calculations are performed so far. Figure 1 shows our current expectation of the QCD phase diagram.

In the study of the QCD phase diagram, the confinement-deconfinement transition plays a crucial role. However, our current understanding of the deconfinement transition is very limited. At least in the heavy quark mass limit, the Polyako-loop which is the gauge invariant holonomy for the imaginary time circle becomes the exact order parameter of the deconfinement transition because the center $\left(\mathbb{Z}_{3}\right)$ symmetry is directly related with the decofinement transition in this case. Although, the Polyakov-loop is no longer the exact order parameter in the finite quark mass regime, the Polyakov-loop is usually treated as the approximate order parameter of the deconfinement transition, and the QCD phase diagra is drown.

In this talk, we discuss the new determination of the deconfinement (pseudo) critical temperature by using the analogy of the topological order which is explained in Sec. 2. Also, we shows the expected QCD phase diagrams at complex chemical potential because we must extend the chemical potential to complex from real when we consider finite $\mu_{\mathrm{R}}$ region in our present determination of the deconfinement (pseudo) critical temperature.

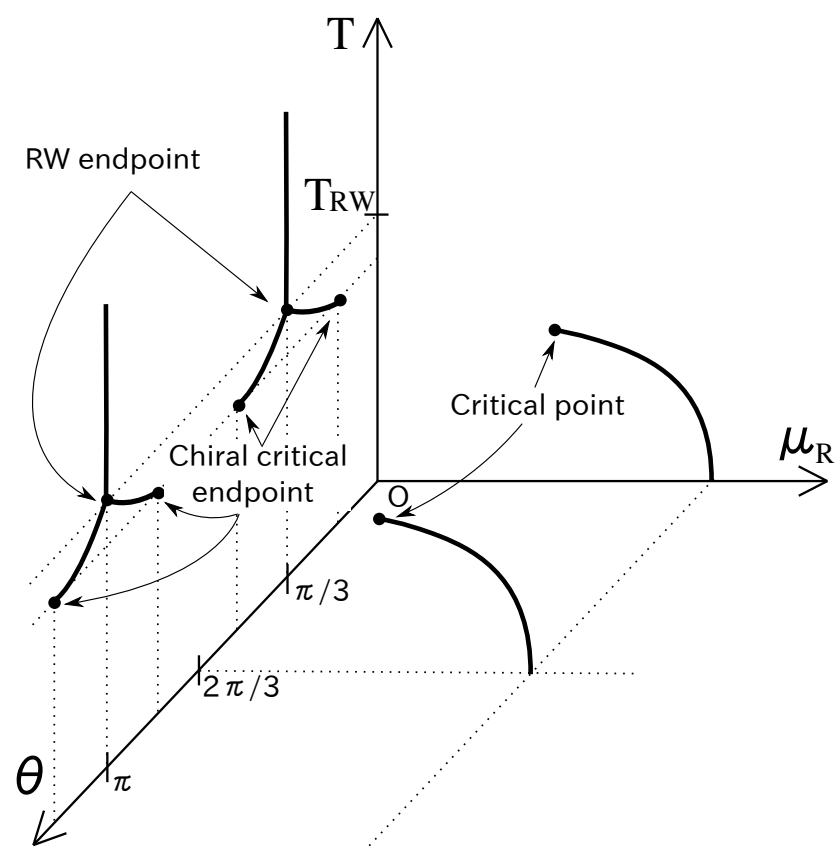

Figure 1: Schematic figure of our current expectation of the QCD phase diagram at finite $\mu_{\mathrm{R}}$ and $\mu_{\mathrm{I}}$, respectively. Solid lines represent the first order transition line. 


\section{Free energy degeneracy and deconfinement transition}

In the imaginary-time formalism, the $U(1)$ flux can be inserted to the closed loop of the imaginary-time direction and then the vector potential is induced by the $U(1)$ flux. Since the appearance form of the vector potential and the imaginary chemical potential is similar in the action, the imaginary chemical potential can be interpreted as the Aharonov-Bohm phase [1]. In this interpretation, we may use the discussion of the topological order discussed in the condensed matter physics [2]. It should be noted that actual applications to zero temperature QCD was already discussed in Ref. [3].

In Ref. [3], the author consider the torus $T^{3}$ at zero temperature. There are introduce three adiabatic operations: (a) Insert the $U(1)$ flux to spatial closed loops, (b) exchange $i$-th and $i+1$-th quarks and (c) move a quark along loops. Commutation relations of the operation (b) and (c) follw the Braid group, and the Aharonov-Bohm effect determines the commutation relations of those operations with operation (a). If quarks are deconfined, above operations become non-commutable because quarks have the fractional charge. On the other hand, commutation relation is commutable if quarks are confined because physical states are characterized by hadron degrees of freedom with integer charges. Therefore, if there is only one vacuum in the deconfined phase, it is inconsistent with the non-commutability of the operations and thus vacuum degeneracy should exist.

At finite $T$, the topological order cannot be well defined, because thermal states are constructed by a mixture of pure states with the Boltzmann factor, and then we cannot operate (a), (b) and (c), adiabatically. Nevertheless, the RW periodicity shows significantly different behaviors in the confined and deconfined phases ${ }^{1}$. It is induced by the nontrivial appearance of the RW periodicity in the deconfined phase. This fact suggests that we can distinguish the confined and deconfined phases at $\mu_{\mathrm{I}}=0$ from the non-trivial free energy degeneracy of the effective potential at $\theta=\pi / 3$. Actually, we can have degenerated free energy minima at $\theta=\pi / 3$ above $T_{\mathrm{RW}}$, but not below $T_{\mathrm{RW}}$. This degeneracy seems similar to the vacuum degeneracy in zero $T$ systems used in the topological order and the analogy can be found; the response of hidden local minima by the flux insertion via $\mu_{\mathrm{I}}$ tells us the non-trivial degeneracy of the free energy minima. Therefore, we propose that $T_{\mathrm{RW}}$ is the pseudo-critical temperature of the confinement-deconfinement transition.

It should be noted that the present definition and the standard definition determined by using the Polyakov-loop are consistent in the infinite quark mass limit where the Polyakov-loop is the exact order-parameter of the confinement-deconfinement transition. Therefore, we can find the relation $T_{\mathrm{D}}=T_{\Phi}=T_{\mathrm{RW}}$ where $T_{\Phi}$ is the critical temperature determined by the susceptibility of the Polyakov-loop and $T_{\mathrm{D}}$ is the deconfinement critical temperature. When the dynamical quarks are taken into account, $T_{\Phi}$ changed to the pseudo-critical temperature. The upper bound of the pseudo-critical temperature may be determined by the appearance of local minima of the effective potential at $\mu_{\mathrm{I}}=0$ in the $\operatorname{Re} \Phi-\operatorname{Im} \Phi$ plane. We call it $T_{\mathbb{Z}_{3}}$. From the lattice QCD and effective model calculations, we can have the relation $T_{\Phi} \leq T_{\mathrm{RW}} \leq T_{\mathbb{Z}_{3}}$. The Polyakov-loop is no longer the exact order parameter and thus the determination of $T_{\mathrm{D}}$ is not unique, but $T_{\mathrm{RW}}$ is uniquely determined. Thus, $T_{\mathrm{RW}}$ which agrees with $T_{\mathrm{D}}$ in the infinite quark mass limit is unambiguously determined in the lattice QCD and effective models and provides a reasonable value as $T_{\mathrm{D}}$ in the

\footnotetext{
${ }^{1}$ For example, this fact can be seen from the comparison between the perturbative one-loop calculation $[4,5]$ and the strong coupling limit of QCD with the mean field approximation $[6,7]$ at finite imaginary chemical potential.
} 
case with the dynamical quark. If we adopt $T_{\mathrm{RW}}$ as $T_{\mathrm{D}}$, we can say that the deconfinement transition is the topological phase transition.

\section{QCD phase diagram at complex chemical potential}

We now discuss the $\mu_{\mathrm{R}}$-dependence of the deconfinement pseudo-critical temperature defined by $T_{\mathrm{RW}}$. We here give an model independent argument based on the perturbative expansion of the effective potential at finite $\mu_{\mathrm{R}}$ as a first step to investigate the $\mu_{\mathrm{R}}$-dependence of the RW endpoint. It should be noted that non-perturbative model approaches have several difficulties. One of the promising effective models is the Polyakov-loop extended Nambu-Jona-Lasinio (PNJL) model [8], but the PNJL model also has the model sign problem at finite $\mu_{\mathrm{R}}$ [9]. There are some proposals to circumvent the model sign problem, for example the complex integral path contour method [10, $11,12]$ based on the Lefschetz thimble $[13,14,15]$ and the complex Langevin method $[16,17]$. Unfortunately those approaches cannot be directly used at finite complex chemical potential at the present because we cannot maintain the RW periodicity and some other desirable properties of QCD.

The effective potential with small $\mu_{\mathrm{R}}$ can be expanded up to $\mu_{\mathrm{R}}^{2}$ order as

$$
\begin{aligned}
\mathscr{V}\left(T, \mu_{\mathrm{R}}, \mu_{\mathrm{I}}\right) & =\mathscr{V}\left(T, 0, \mu_{\mathrm{I}}\right)-\left(\frac{\mu_{\mathrm{R}}}{T}\right)\left(\left.\operatorname{Tn}_{q}\left(\mu_{\mathrm{R}}, \mu_{\mathrm{I}}\right)\right|_{\mu_{\mathrm{R}}=0}\right) \\
& -\left.\frac{1}{2}\left(\frac{\mu_{\mathrm{R}}}{T}\right)^{2} \frac{d\left[\operatorname{Tn}_{q}\left(\mu_{\mathrm{R}}, \mu_{\mathrm{I}}\right)\right]}{d \mu_{\mathrm{R}} / T}\right|_{\mu_{\mathrm{R}}=0}+\mathscr{O}\left(\left(\mu_{\mathrm{R}} / T\right)^{3}\right),
\end{aligned}
$$

where

$$
\left.T \frac{d n_{q}}{d \mu_{\mathrm{R}} / T}\right|_{\mu_{\mathrm{R}}=0}=\left.T \frac{d n_{q}}{d\left(i \mu_{\mathrm{I}} / T\right)}\right|_{\mu_{\mathrm{R}}=0}=-\left.i T \frac{d n_{q}}{d \theta}\right|_{\mu_{\mathrm{R}}=0} .
$$

Equation (3.2) is real. The second term in r.h.s. of Eq. (3.1) is pure imaginary.

Here, we consider the confinement and deconfinement configurations. We call the configuration at $\left(T_{\mathrm{RW}}-\varepsilon\right)$ confinement configuration which is labeled as $\mathscr{C}_{-\varepsilon}$ where $\varepsilon$ is a infinitesimal real positive value. Also, we call the configuration at $\left(T_{\mathrm{RW}}+\varepsilon\right)$ deconfinement configuration which is labeled as $\mathscr{C}_{+\varepsilon}$. By comparing $\operatorname{Re} \mathscr{V}$ with $\mathscr{C}_{-\varepsilon}$ to that with $\mathscr{C}_{+\varepsilon}$ in the $\varepsilon \rightarrow 0$ limit, we can distinguish whether $T_{\mathrm{RW}}$ decreases or increases if we can estimate the third term of r.h.s. of Eq. (3.1). From the lattice QCD and effective model calculations [18, 19], we can estimate that Eq. (3.2) is negative below $T_{\mathrm{RW}}$ and it becomes moderate above $T_{\mathrm{RW}}$. The $\mu_{\mathrm{R}}^{2}$ correction term makes Re $\mathscr{V}$ with $\mathscr{C}_{-\varepsilon}$ higher than that with $C_{+\varepsilon}$ because the $\mu_{\mathrm{R}}^{2}$ correction term is then positive with $\mathscr{C}_{+\varepsilon}$. This fact means that the first-order $T_{\mathrm{RW}}$ decreases with increasing $\mu_{\mathrm{R}}$ at least in the small $\mu_{\mathrm{R}}$ region. This behavior is consistent with the decreasing behavior of the pseudo-critical temperature of the deconfinement transition defined by using usual determinations which consider the Polyakov-loop; for example, see Refs [8, 20, 21].

By taking into account our perturbative result and the symmetry argument, we can sketch expected QCD phase diagrams at finite complex chemical potential. QCD phase diagrams expected from the symmetry argument and our present perturbative calculations are summarized in Fig. 2. Because of the RW periodicity, the phase structure should be periodic along the $\theta$ axis. The RW transition line on the $(T, \theta)$ plane $\left(\mu_{\mathrm{R}}=0\right)$ may be connected with the first order phase transition boundary on the $\left(T, \mu_{\mathrm{R}}\right)$ plane. Two of the first order transition lines starting from the RW endpoint 

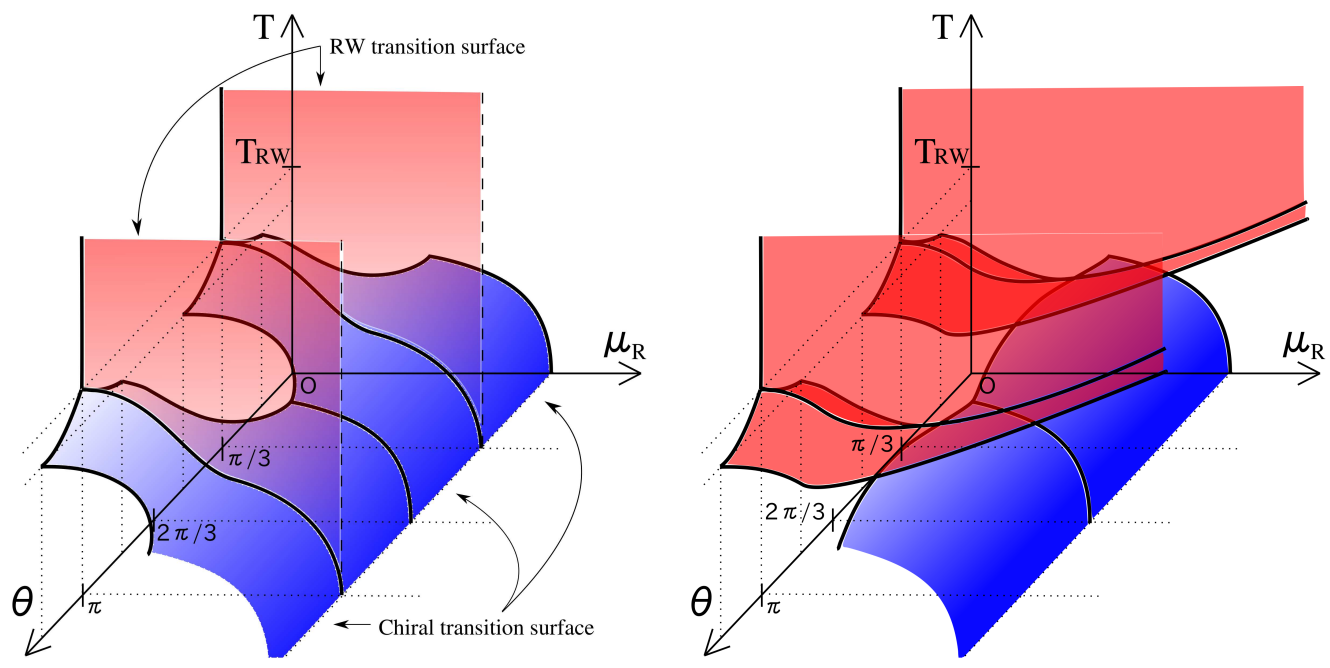

Figure 2: Two possible schematic QCD phase diagrams at finite complex chemical potential. The left (right) panel represents the correlated (uncorrelated) case between the chiral and RW transition surfaces.

have chiral transition natures and are referred to as the chiral transition lines [22]. Then, it is not unreasonable to expect that the endpoint of the chiral critical line on the $(T, \theta)$ plane is connected with the critical point on the $\left(T, \mu_{\mathrm{R}}\right)$ plane. In this case, the first order phase boundary on the $\left(T, \mu_{\mathrm{R}}\right)$ plane forms a chiral transition surface in the $\left(T, \mu_{\mathrm{R}}, \theta\right)$ space, and connects the $\left(T, \mu_{\mathrm{R}}\right)$ plane and the $(T, \theta)$ plane. The RW transition line extends in the finite $\mu_{\mathrm{R}}$ region and forms an RW transition surface in the $\left(T, \mu_{\mathrm{R}}, \theta\right)$ space. The RW endpoint may reach $T=0$ as shown in the top panel of Fig. 2 or it may deviate from the chiral transition surface at some temperature. There is the possibility that $T_{\mathrm{RW}}$ line becomes smaller than the chiral critical surface at moderate $\mu_{\mathrm{R}}$ and finally becomes larger than the chiral transition surface.. It is deeply related with a strength of the correlation between the chiral transition surface and the RW transition surface.

Another possibility is that the first order transition lines on the $(T, \theta)$ plane are separated from the first order phase boundary on the $\left(T, \mu_{\mathrm{R}}\right)$ plane, as shown in the bottom panel of Fig. 2. $T_{\mathrm{RW}}$ first decreases at small $\mu_{\mathrm{R}}$, but does not goes across the chiral transition surface. In this case, the deconfinement transition represented by the RW endpoint on the $(T, \theta)$ plane has less relevance to the first order phase boundary, which would be the chiral transition, on the $\left(T, \mu_{\mathrm{R}}\right)$ plane. Therefore, we can call this possibility uncorrelated case and the former possibility correlated case.

The QCD phase diagram at finite complex chemical potential may be related with the following subjects. (I) In Ref. [23, 24], the authors use experimental data to construct the canonical partition function. Then, $T_{\mathrm{RW}}$ at $\mu_{\mathrm{R}}=0$ is used to clarify realized temperatures in experiments through the Lee-Yang zero analysis [25, 26]. In the analysis, $T_{\mathrm{RW}}$ at finite $\mu_{\mathrm{R}}$ should be related with zeros inside the unit circle on the complex quark fugacity plane. If so, there is the possibility that we can strictly determine realized temperatures in experiments if we can systematically understand the behavior of zeros. (II) The analytic continuation in QCD from the imaginary to the real chemical potential is usually performed on the $\mu^{2}$ plane. In the continuation, we may miss some information such as an inhomogeneous chiral condensate [27]. The analytic continuation on the 
complex chemical potential plane may restore such information missing.

\section{Summary}

We have proposed that the Roberge-Weiss endpoint provides a reasonable deconfinement temperature. The imaginary chemical potential can be interpreted as the Aharonov-Bohm phase induced by $U(1)$ flux insertions and then the analogy of the topological order can be found. In the deconfined phase, we can find the degeneracy free energy degeneracy, but we cannot find such nontrivial structure of the free energy in the confined phase. This suggests that we can distinguish the confined and deconfined phases at $\theta=\mu_{\mathrm{I}} / T=0$ from the appearance of the non-trivial degeneracy of the effective potential. Then, $T_{\mathrm{RW}}$ can be considered as the pseudo-critical temperature at $\mu=0$.

In this study, we have used the analogy of the topological order at finite $T$. The topological transition does not have the usual order parameter, but the relation with an entanglement and topological entropies has been discussed in the condensed matter physics; for example, see Ref. [28]. In QCD, such a relation is not clear, but it is an interesting direction to investigate the topological nature of the transition.

Using the perturbative expansion in terms of $\mu_{\mathrm{R}}$ of the effective ptoential, we have investigated the behavior of $T_{\mathrm{RW}}$ at small $\mu_{\mathrm{R}}$ and then the decreasing behavior of $T_{\mathrm{RW}}$ is obtained. Based on these results, we presented two scenarios of the QCD phase diagram at finite complex chemical potential. First scenario has the strong correlation between the chiral and deconfinement transitions. The RW endpoint at finite $\mu_{\mathrm{R}}$ finally reaches the $T=0$ point. Then, the critical point can become more complex than the usual expectation since two more first order transition lines are connected at the critical point. The second scenario is the uncorrelated case and then the RW endpoint is separated from the chiral transition surface.

Since the complex chemical potential is related with the Lee-Yang zero analysis and also the analytic continuation to the finite $\mu_{\mathrm{R}}$ region, understanding the QCD phase diagram at finite complex chemical potential may be important to the beam energy scan program in heavy ion collider experiments, investigation of neutron star structures and so on.

\section{References}

[1] Y. Aharonov and D. Bohm. Significance of electromagnetic potentials in the quantum theory. Phys.Rev., 115:485-491, 1959.

[2] X.G. Wen. Topological Order in Rigid States. Int.J.Mod.Phys., B4:239, 1990.

[3] Masatoshi Sato. Topological discrete algebra, ground state degeneracy, and quark confinement in QCD. Phys.Rev., D77:045013, 2008.

[4] David J. Gross, Robert D. Pisarski, and Laurence G. Yaffe. QCD and Instantons at Finite Temperature. Rev.Mod.Phys., 53:43, 1981.

[5] Nathan Weiss. The Effective Potential for the Order Parameter of Gauge Theories at Finite Temperature. Phys.Rev., D24:475, 1981.

[6] Yusuke Nishida. Phase structures of strong coupling lattice QCD with finite baryon and isospin density. Phys.Rev., D69:094501, 2004. 
[7] N. Kawamoto, K. Miura, A. Ohnishi, and T. Ohnuma. Phase diagram at finite temperature and quark density in the strong coupling limit of lattice QCD for color SU(3). Phys.Rev., D75:014502, 2007.

[8] Kenji Fukushima. Chiral effective model with the Polyakov loop. Phys.Lett., B591:277-284, 2004.

[9] Kenji Fukushima and Yoshimasa Hidaka. A Model study of the sign problem in the mean-field approximation. Phys.Rev., D75:036002, 2007.

[10] Hiromichi Nishimura, Michael C. Ogilvie, and Kamal Pangeni. Complex saddle points in QCD at finite temperature and density. 2014.

[11] Hiromichi Nishimura, Michael C. Ogilvie, and Kamal Pangeni. Complex Saddle Points and Disorder Lines in QCD at finite temperature and density. 2014.

[12] Yuya Tanizaki, Hiromichi Nishimura, and Kouji Kashiwa. Evading the sign problem in the mean-field approximation through Lefschetz-thimble path integral. 2015.

[13] Edward Witten. Analytic Continuation Of Chern-Simons Theory. pages 347-446, 2010.

[14] Marco Cristoforetti, Francesco Di Renzo, and Luigi Scorzato. New approach to the sign problem in quantum field theories: High density QCD on a Lefschetz thimble. Phys.Rev., D86:074506, 2012.

[15] H. Fujii, D. Honda, M. Kato, Y. Kikukawa, S. Komatsu, et al. Hybrid Monte Carlo on Lefschetz thimbles - A study of the residual sign problem. JHEP, 1310:147, 2013.

[16] G. Parisi and Yong-shi Wu. Perturbation Theory Without Gauge Fixing. Sci.Sin., 24:483, 1981.

[17] G. Parisi. ON COMPLEX PROBABILITIES. Phys.Lett., B131:393-395, 1983.

[18] Massimo D’Elia and Maria-Paola Lombardo. Finite density QCD via imaginary chemical potential. Phys.Rev., D67:014505, 2003.

[19] Yuji Sakai, Kouji Kashiwa, Hiroaki Kouno, and Masanobu Yahiro. Phase diagram in the imaginary chemical potential region and extended Z(3) symmetry. Phys.Rev., D78:036001, 2008.

[20] C. Sasaki, B. Friman, and K. Redlich. Susceptibilities and the Phase Structure of a Chiral Model with Polyakov Loops. Phys.Rev., D75:074013, 2007.

[21] Kouji Kashiwa, Hiroaki Kouno, Masayuki Matsuzaki, and Masanobu Yahiro. Critical endpoint in the Polyakov-loop extended NJL model. Phys.Lett., B662:26-32, 2008.

[22] Massimo D'Elia and Francesco Sanfilippo. The Order of the Roberge-Weiss endpoint (finite size transition) in QCD. Phys.Rev., D80:111501, 2009.

[23] Atsushi Nakamura and Keitaro Nagata. Probing QCD Phase Structure by Baryon Multiplicity Distribution. 2013.

[24] Keitaro Nagata, Kouji Kashiwa, Atsushi Nakamura, and Shinsuke M. Nishigaki. Lee-Yang zero distribution of high temperature QCD and Roberge-Weiss phase transition. 2014.

[25] Chen-Ning Yang and T.D. Lee. Statistical theory of equations of state and phase transitions. 1. Theory of condensation. Phys.Rev., 87:404-409, 1952.

[26] T.D. Lee and Chen-Ning Yang. Statistical theory of equations of state and phase transitions. 2. Lattice gas and Ising model. Phys.Rev., 87:410-419, 1952.

[27] Kouji Kashiwa, Tong-Gyu Lee, Kazuya Nishiyama, and Ryo Yoshiike. Inhomogeneous chiral condensates and non-analyticity under an external magnetic field. 2015.

[28] Michael Levin and Xiao-Gang Wen. Detecting Topological Order in a Ground State Wave Function. Phys. Rev. Lett., 96:110405, 2006. 